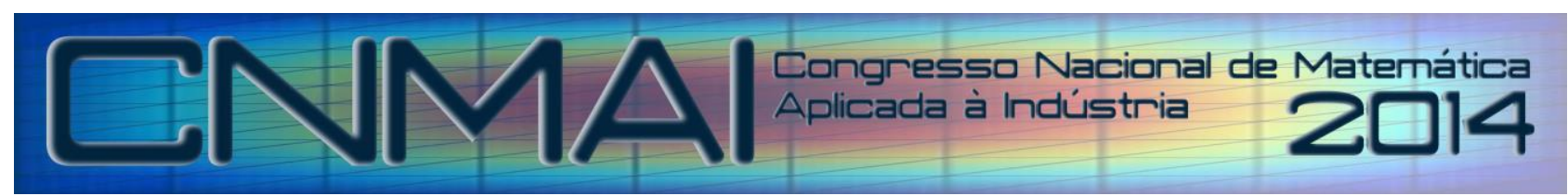

18 a 21 de novembro de 2014, Caldas Novas - Goiás

\title{
Estudo Numérico-computacional do Método da Entropia Máxima na Análise de Incertezas Paramétricas de Sistemas em Engenharia
}

\author{
Marcelo Henrique Belonsi - marcelobelonsi@bol.com.br ${ }^{1}$ \\ Antonio Marcos Gonçalves de Lima - amglima@ mecanica.ufu.br ${ }^{2}$ \\ Romer Gonçalves - romer.engenharia@gmail.com²
}

\begin{abstract}
${ }^{1}$ Universidade Estadual de Goiás, Departamento de Matemática, Rua 14, 625, Jardim América, Morrinhos-GO.
${ }^{2}$ Universidade Federal de Uberlândia, Faculdade de Engenharia Mecânica, João Naves de Ávila, 2121, Santa Monica, Uberlândia-MG.
\end{abstract}

\begin{abstract}
Resumo: O Princípio da Máxima Entropia teve seu desenvolvimento entre os anos de 1844 a 1906, com a publicaçao dos resultados obtidos com a Equação do Transporte de Boltzmann e o Teorema H de Boltzmann, cujos objetivos eram determinar a variação temporal de uma função de distribuição de probabilidades, além da caracterização de uma métrica para a Equação do Transporte de Boltzmann. Entretanto, vendo a possibilidade de estender o conceito de entropia para sistemas físicos, Shannon empregou os desenvolvimentos feitos por Boltzmann para fins de utilização na teoria da informação na busca de uma medida de entropia, que caracterizasse a distribuição de probabilidade de uma variável aleatória. Neste sentido, o presente trabalho é dedicado à formalização teórica sobre o Princípio e suas propriedades a partir do trabalho de Shannon, visando caracterizar suas fórmulas no sentido de demonstrar sua robustez e eficácia na caracterização das densidades de probabilidades em aplicações em sistemas de engenharia. Assim, será evidenciado todos os desenvolvimentos teóricos para a caracterização das relações matemáticas essenciais na utilização do Princípio da Máxima Entropia, com vistas à sua aplicabilidade aos sistemas dinâmicos em geral e mostrar como a matemática da probabilidade se faz presente nas mais diversas aplicações, especialmente, nas aplicações em engenharia mecânica.
\end{abstract}

Palavras-chave: Quantificação de incertezas; Princípio da Máxima Entropia; Matemática; Dinâmica.

\section{INTRODUÇÃO}

No contexto dos novos desenvolvimentos em vários domínios da engenharia mecânica, nota-se a constante busca pela durabilidade, confiabilidade, segurança e conforto de máquinas, produtos e equipamentos impulsionados pela modernização de processos e produtos industriais. Além disso, normas ambientais cada vez mais severas impõem limites restritos para os níveis de operação, de segurança e de conforto de produtos e serviços, que estão frequentemente presentes em vários ramos da atividade produtiva, tais como as indústrias de construção civil, automotiva, aeroespacial, naval, de telecomunicações, mineração, metalúrgica, siderúrgica, de geração e distribuição de energia, etc. Neste panorama, a importância dada á análise de confiabilidade estrutural de sistemas mecânicos é hoje um dos problemas cruciais da Engenharia Mecânica, em virtude da tendência de realização de estruturas cada vez mais extensas e leves, e de aumento considerável da velocidade de operação das máquinas. Por exemplo, as vibrações e o ruído de aeronaves tem sido a maior causa do desconforto de passageiros e usuários, e este problema é agravado com o uso progressivo de materiais compostos e motores mais potentes como ilustrado na Fig. 1, que representa a superfície de parte de um componente de aeronave que falhou por fadiga quando submetida a cargas cíclicas (Koçer, 2010).

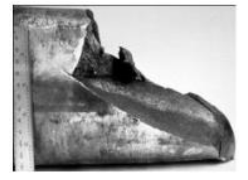

Figura 1: Foto de parte de uma superfície de aeronave que falhou por fadiga (Koçer, 2010). 
Outro aspecto importante que deve ser levado em conta quando se trata de confiabilidade estrutural é a modelagem estocástica e a quantificação das incertezas paramétricas presentes na estrutura dinâmica (Lima et al., 2010; Sampaio e Cataldo, 2010). Acrescenta-se a isto o fato de que o uso de técnicas de otimização multiobjetivas que buscam os valores ótimos das funções objetivos originais ao mesmo tempo em que as funções que caracterizam as dispersões nos parâmetros de projeto, são imprescindíveis para a obtenção de regiões "confiáveis ótimas e robustas" de acordo com o critério de falha empregado na análise (Lambert et al., 2010). Dessa forma, o presente trabalho visa apresentar um estudo matemático e implementação computacional do Método da Máxima Entropia para a caracterização das verdadeiras distribuições de probabilidades de variáveis aleatórias com uma aplicação simples em um sistema dinâmico discreto.

\section{MÉTODO DA MÁXIMA ENTROPIA}

O início dos estudos utilizando Entropia se deu, principalmente, com o desenvolvimento dos trabalhos de Boltzmann ${ }^{1}$ a partir do século IXX, mais especificamente, a partir dos resultados obtidos com a Equação do Transporte de Boltzmann (ETB) e do o Teorema H de Boltzmann para determinar a variação temporal de uma distribuição de probabilidade e uma métrica H que satisfaça a ETB (Cattani e Bassalo, 2008). Dessa forma, pode-se observar que ambos os trabalhos estão correlacionados e seus resultados utilizam o conceito de Entropia. Assim, partindo-se de um vetor de variáveis aleatórias $\mathrm{X}=\left\{\mathrm{x}_{1}, \mathrm{x}_{2}, \ldots, \mathrm{x}_{\mathrm{n}}\right\}$, nas quais possuem as seguintes probabilidades associadas $\left(\mathrm{p}_{1}, \mathrm{p}_{2}, \ldots\right.$, $\left.\mathrm{p}_{\mathrm{n}}\right)$, de forma que $\mathrm{p}_{1}+\mathrm{p}_{2}+\mathrm{p}_{3}+\ldots+\mathrm{p}_{\mathrm{n}}=1$, o interesse é encontrar uma quantidade $\mathrm{H}\left(\mathrm{p}_{1}, \ldots, \mathrm{p}_{\mathrm{n}}\right)$, que meça de forma única a quantidade de incerteza associada a esta distribuição de probabilidade. Em outras palavras, deseja-se encontrar uma quantidade $\mathrm{H}\left(\mathrm{p}_{1}, \ldots, \mathrm{p}_{\mathrm{n}}\right)$ que determine de maneira única a densidade de probabilidade da distribuição dada. Assim, para que se possa encontrar uma medida que seja capaz de determinar a densidade de probabilidade e, de forma consequente caracterizar tal distribuição, esta deverá obedecer às três condições básicas, definidas por Shannon (1948):

i. H deve ser uma função contínua em $\mathrm{p}_{\mathrm{i}}$;

ii. Se todos os $\mathrm{p}_{\text {is }}$ são iguais, a quantidade $\mathrm{A}(\mathrm{n})=\mathrm{H}(1 / \mathrm{n}, \ldots, 1 / \mathrm{n})$ é uma função monótona crescente em $\mathrm{n}$;

iii. Se uma escolha pode ser dividida entre duas escolhas sucessivas, o H original deve ser a soma ponderada dos valores individuais de $\mathrm{H}$.

A lei de composição em vez de expressar as probabilidades dos eventos $\left(\mathrm{n}_{1} \ldots, \mathrm{n}_{\mathrm{n}}\right)$ diretamente, esta utiliza-se de uma construção de forma para a obtenção de seqüências de funções monótonas crescentes. Assim, de acordo com Jaynes (1957) sempre é possível obter a incerteza, independentemente como as escolhas são agrupadas, em outras palavras, o processo para obter a quantidade $\mathrm{H}$ é um processo construtivo e, como tal este não se pauta pela unicidade no desenvolvimento do processo, mas sim, no respeito às regras de construção definidas por Shannon. Para proceder tal transformação para se obter esta seqüência, deve-se agrupar os primeiros k elementos do conjunto $\mathrm{X}$ de forma que estes formem um único evento, com sua probabilidade igual a $\mathrm{w}_{1}=\mathrm{p} 1+\mathrm{p} 2+\ldots+\mathrm{pk}$ então o próximos $\mathrm{n}-\mathrm{k}$ possíveis valores podem serão agrupados de forma a se obter um segundo evento com probabilidade $\mathrm{w}_{2}=\mathrm{p}_{\mathrm{k}+1}+\ldots+\mathrm{p}_{\mathrm{n}} \mathrm{e}$, assim sucessivamente.

A titulo de exemplo, pode citar o caso em que se agrupam o conjunto $\mathrm{X}$ em dois eventos com probabilidades iguais a $\mathrm{w}_{1}$ e $\mathrm{w}_{2}$, respectivamente:

$$
\underbrace{\mathrm{n}_{1}, \mathrm{n}_{2}, \mathrm{n}_{3}, \ldots, \mathrm{n}_{\mathrm{k}}}_{\mathrm{w}_{1}=\mathrm{p}_{1}+\mathrm{p}_{2}+\ldots+\mathrm{p}_{\mathrm{k}}}
$$

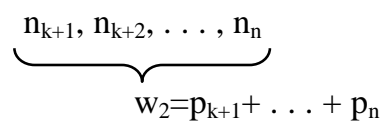

Nesse sentido, o grau de incerteza quanto aos eventos compostos é $\mathrm{H}\left(\mathrm{w}_{1}, \mathrm{w}_{2}\right)$. Assim, pode-se escrever a medida, $\mathrm{H}$, em termos das probabilidades condicionais $\left(\mathrm{p}_{1} / \mathrm{w}_{1}, \ldots, \mathrm{p}_{\mathrm{k}} / \mathrm{w}_{1}\right)$ para representar a quantidade $\mathrm{H}$ relacionadas aos eventos finais $\left(\mathrm{n}_{1}, \ldots, \mathrm{n}_{\mathrm{k}}\right)$ que dão o primeiro evento composto como se ele tivesse ocorrido. A partir dessa construção chega-se, finalmente, ao mesmo estado do conhecimento, como se $\left(\mathrm{p}_{1}, \ldots, \mathrm{p}_{\mathrm{n}}\right)$ tenha sido dado diretamente, pois, para que a medida de informação seja consistente, tal medida deve obter a mesma incerteza final não importa o quão as escolhas sejam realizadas. Assim, devemos ter:

$$
H\left(p_{1}, \ldots, p_{n}\right)=H\left(w_{1}, w_{2}\right)+w_{1} H\left(\frac{p_{1}}{w_{1}}, \ldots, \frac{p_{k}}{w_{1}}\right)+w_{2} H\left(\frac{p_{k+1}}{w_{2}}, \ldots, \frac{p_{n}}{w_{2}}\right) \ldots
$$

Utilizando os resultados obtidos por Jaynes (1957), é possível escrever a entropia de qualquer variável aleatória $\mathrm{N}=\left\{\mathrm{n}_{\mathrm{i}}\right\}$ com probabilidades associadas $\mathrm{p}_{\mathrm{i}},(\mathrm{i}=1,2,3, \ldots, \mathrm{n})$. da seguinte forma:

$$
A\left(\sum \mathrm{n}_{\mathrm{i}}\right)=H\left(p_{1}, p_{2}, \ldots, p_{n}\right)+\sum p_{i} \mathrm{~A}\left(\mathrm{n}_{\mathrm{i}}\right)
$$

\footnotetext{
${ }^{1}$ Ludwig Eduard Boltzmann (1844 -1906) foi um físico austríaco, conhecido pelo seu trabalho nos campo da termodinâmica estatística. É considerado junto com Josiah Willard Gibbs e James Clerk Maxwell como o fundador da mecânica estatística.
} 
Portanto, considerando a Eq. (2) e, ainda, $\mathrm{n}_{\mathrm{i}}=\mathrm{m}$, escreve-se:

$$
\begin{gathered}
A(n . m)=A(n)+n \sum \frac{1}{n} \mathrm{~A}(\mathrm{~m}) \\
A(n . m)=A(n)+\mathrm{A}(\mathrm{m})
\end{gathered}
$$

Na Eq. (3), A(n) deve ser uma função que tenha a propriedade de transformar produto em soma, o que leva a considerar que esta função pode ser expressa pela função logarítmica. Dessa forma, fica evidente uma das razões que levou Shannon a expressar a medida de entropia por meio da função logarítmica. Como desdobramento, é possível enunciar o seguinte resultado:

Uma medida, H, que satisfaz as três propriedades definidas por Shannon (1948) é da forma:

$$
H=-k \sum_{i=1}^{n} p_{i} \ln \left(p_{i}\right) \quad \text { onde } k>0
$$

Como está sendo considerado neste trabalho eventos com chances reais de ocorrência, excetuando-se o caso do evento certo, termos que $0<p_{i}<1$, para $\mathrm{i}=1,2,3, \ldots, \mathrm{n}$. Assim:

$$
\lim _{x \rightarrow p_{i}} H=-k \sum_{i=1}^{n} \lim _{x \rightarrow p_{i}} x \ln (x)=-k \sum_{i=1}^{n} p_{i} \ln \left(p_{i}\right)
$$

Logo H é contínua em pi. Considerando, ainda, $0<p_{i}<1$, tem-se:

$$
A(n)=H\left(\frac{1}{n}, \ldots, \frac{1}{n}\right)=-k \sum_{i=1}^{n} \frac{1}{n} \ln \left(\frac{1}{n}\right)=k \ln (n)
$$

Assim, A(n) é uma função monótona crescente, pois $A^{\prime}(n)=k / n>0$. Da condição (i), é suficiente para determinar $\mathrm{H}$ para todos os valores racionais,

$$
p_{i}=\frac{n_{i}}{\sum_{i=1}^{n} n_{i}}
$$

A condição (iii) significa que $\mathrm{H}$ já está determinada a partir das quantidades simetrias A(n). Para considerar uma escolha de uma das alternativas $\left(\mathrm{x}_{1}, \ldots \mathrm{x}_{\mathrm{n}}\right)$, como um primeiro passo na escolha de uma das alternativas igualmente prováveis. A segunda etapa do qual é também uma escolha entre $\mathrm{n}_{\mathrm{i}}$ alternativas igualmente prováveis. Em geral, isto pode ser escrito, pela Eq. (5).

$$
H\left(p_{1}, \ldots, p_{n}\right)+\sum_{i=1}^{n} p_{i} A\left(n_{i}\right)=A\left(\sum_{i=1}^{n} n_{i}\right)
$$

Em particular, pode-se escolher todos os elementos $n_{i}$ iguais a m. Assim, $p_{i}=1 / n$ e, a Eq. (5) se reduz a:

$$
H\left(\frac{1}{n}, \ldots, \frac{1}{n}\right)+\sum_{i=1}^{n} \frac{1}{n} A\left(n_{i}\right)=A\left(\sum_{i=1}^{n} n_{i}\right)
$$

Logo, de acordo com a condição (ii) tem-se que $\mathrm{A}(\mathrm{n})=\mathrm{H}(1 / \mathrm{n}, \ldots, 1 / \mathrm{n})$

$$
A(n)+A(m)=A(n \cdot m)
$$

Nota-se nesta última equação a propriedade de transformar uma soma em produto, assim como já vimos na Eq. (3). Do mesmo modo, uma escolha adequada para a solução da equação acima será expressar a função $\mathrm{A}(\mathrm{n})$ em termos de uma função logarítmica, da forma:

$$
A(n)=k \ln (n)
$$

Doravante, substituindo a Eq. (6) na Eq. (5), escreve-se: 


$$
\begin{gathered}
H\left(p_{1}, \ldots, p_{n}\right)+\sum_{i=1}^{n} p_{i} k \ln \left(n_{i}\right)=k \ln \left(\sum_{i=1}^{n} n_{i}\right) \\
H\left(p_{1}, \ldots, p_{n}\right)=-k\left[-\ln \left(\sum_{i=1}^{n} n_{i}\right)+\sum_{i=1}^{n} \ln \left(n_{i} p_{i}\right)\right] \\
H\left(p_{1}, \ldots, p_{n}\right)=-k\left[-\ln \left(\sum_{i=1}^{n} n_{i}\right)+\ln \left(\prod_{i=1}^{n} n_{i} p_{i}\right)\right] \\
H\left(p_{1}, \ldots, p_{n}\right)=-k\left[\sum_{i=1}^{n} p_{i} \ln \left(\frac{n_{i}}{\sum n_{i}}\right)\right]
\end{gathered}
$$

Finalmente, é possível chegar-se à seguinte expressão para a entropia.

$$
H\left(p_{1}, \ldots, p_{n}\right)=-k \sum_{i=1}^{n} p_{i} \ln \left(p_{i}\right)
$$

De forma similar é possível estabelecer a seguinte medida de entropia para uma variável aleatória contínua por meio da expressão:

$$
H=-k \int_{a}^{b} p(x) \ln (x) d x
$$

As medidas dadas pelas Eqs. (7) e (8) são fundamentais na extração da incerteza e desempenham um papel central na teoria da informação. Vale salientar que $\mathrm{H}(\mathrm{x})$ representa a entropia para a variável aleatória $\mathrm{X}$ e, ainda, que a base do logaritmo equivale a simplesmente uma escolha adequada para a constante $\mathrm{k}$ :

$$
H=-k \sum_{i=1}^{n} p_{i} \log _{b}\left(p_{i}\right)=-\frac{k}{\ln (b)} \sum_{i=1}^{n} p_{i} \ln \left(p_{i}\right)
$$

Considerando uma variável aleatória binomial é possível visualizar a partir da Fig. 2 a entropia, $\mathrm{H}(\mathrm{p}, 1-\mathrm{p})$, da variável aleatória, $\mathrm{X}$, considerando dois valores distintos para $\mathrm{k}$. Assim, substituindo os valores $\mathrm{n}=2, \mathrm{p}_{1}=\mathrm{p}$ e $\mathrm{p}_{2}=1-\mathrm{p}$ na Eq (7), escreve-se:

$$
H=-k[p \ln (p)+(1-p) \ln (1-p)]
$$

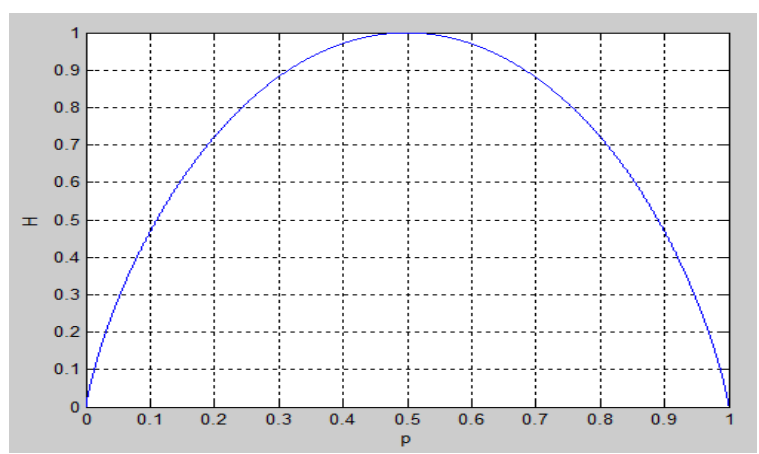

(a) $\mathrm{k}=1 / \ln (2)$

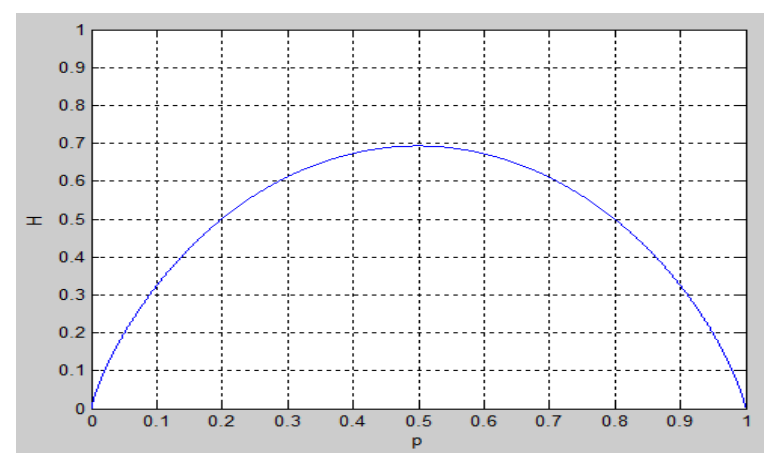

(b) $\mathrm{k}=1$

Figura 2: Entropia H(p,1-p) para dois, diferentes, valores k.

A partir da Fig. 2, é possível observar que a escolha de k influencia apenas no maior valor obtido pela medida $\mathrm{H} \mathrm{e}$, nada influencia na distribuição de probabilidade considerada e, de forma, consequente na distribuição a ser determinada pelo Método da Máxima Entropia. Neste caso, é possível observar a entropia máxima ocorrendo quando os eventos são equiprováveis. Entretanto, é importante destacar que este resultado é muito fácil de ser obtido no caso em que a variável aleatória é binomial, como segue:

$$
H(X)=-k \sum_{i=1}^{2} p_{i} \ln \left(p_{i}\right)=-k[p \ln (p)+(1-p) \ln (1-p)]
$$




$$
\begin{gathered}
\frac{d H(X)}{d p}=-k \frac{d[p \ln (p)+(1-p) \ln (1-p)]}{d p} \\
\frac{d H(X)}{d p}=-k\left[\ln (p)+\frac{1}{p} p-\ln (1-p)+\frac{1}{1-p}(-1)(1-p)\right] \\
\frac{d H(X)}{d p}=-k[\ln (p)+1-\ln (1-p)-1] \\
\frac{d H(X)}{d p}=-k[\ln (p)-\ln (1-p)] \\
\frac{H(X)}{d p}=-k\left[\ln \left(\frac{p}{1-p}\right)\right]=0 \\
\left.\frac{p}{\frac{p}{1-p}}=1 \quad \text { ou } \quad p=\frac{p}{2}\right)=0
\end{gathered}
$$

Pelo teste da derivada segunda tem-se:

$$
\frac{d^{2} H(X)}{d p^{2}}=-k\left[\frac{1}{p}+\frac{1}{1-p}\right] \quad \text { ou } \quad \frac{d^{2} H(X)}{d p^{2}}=-k\left[\frac{1}{p(1-p)}\right]
$$

Como $0<\mathrm{p}<1$

$$
\frac{d^{2} H(X)}{d p^{2}}=-k\left[\frac{1}{p(1-p)}\right]<0
$$

Para todo $0<\mathrm{p}<1$. Logo pelo critério da derivada segunda $\mathrm{p}=1 / 2$ é um ponto de máximo da função $\mathrm{H}(\mathrm{X})$.

Até o momento tratou-se das condições de existência da entropia, $\mathrm{H}$, e da medida de entropia $\mathrm{H}$, associada a uma variável aleatória X. Será agora utilizada esta medida de entropia para fins de caracterização da densidade de probabilidade da variável aleatória $X$. Nesse sentido, seja uma variável aleatória discreta $X=\left\{x_{1}, x_{2}, x_{3}, \ldots, x_{n}\right\}$ tais que $\mathrm{x}_{\mathrm{i}} \neq \mathrm{x}_{\mathrm{j}}$ para $\mathrm{i} \neq \mathrm{j}$. Vale dizer que este procedimento descrito também pode ser utilizado para uma variável aleatória contínua. Assim, sabe-se que existe um conjunto $\mathrm{P}=\left\{\mathrm{p}_{1}, \mathrm{p}_{2}, \mathrm{p}_{3}, \ldots, \mathrm{p}_{\mathrm{n}}\right\}$ de probabilidades associadas a cada um dos elementos de X. Dessa forma, a intenção é encontrar os valores $\mathrm{p}_{\mathrm{i}}$, tais que:

$$
\sum_{i=1}^{n} p_{i}=1 \quad \text { com } 0 \leq p_{i} \leq 1
$$

Que satisfaçam a medida de entropia definida por Shannon

$$
H\left(p_{1}, \ldots, p_{n}\right)=-k \sum_{i=0}^{n} p_{i} \ln \left(p_{i}\right)
$$

Neste mesmo sentido busca-se encontrar os valores de $\mathrm{p}_{1}, \mathrm{p}_{2}, \mathrm{p}_{3}, \ldots, \mathrm{p}_{\mathrm{n}}$, que maximizam a medida $\mathrm{H}$, o que nos coloca à frente de um problema de maximização da medida de entropia, $\mathrm{H}$, restrito ao vínculo definido na Eq. (9). Este problema torna-se relativamente fácil, porém muito trabalhoso, quando utiliza-se o método dos multiplicadores de Lagrange, para resolver este problema. Para melhor facilitar a compreensão e sem perda de generalidade será assumido $\mathrm{k}=1$.

$$
H(p)=-\sum_{i=0}^{n} p_{i} \ln \left(p_{i}\right)
$$

Aplicando o método do multiplicador de Lagrange à medida $\mathrm{H}$ sujeita ao vínculo (9) tem-se:

$$
L\left(p, \lambda_{0}\right)=H(p)-\lambda_{0}\left[\left(\sum_{i=1}^{n} p_{i}\right)-1\right]
$$

Desenvolvendo esta ultima equação, chega-se à seguinte expressão.

$$
p_{i}=e^{-\left(1+\lambda_{0}\right)}=c t e
$$


Portanto observa-se, a partir da Eq. (10) que a variável aleatória possui uma distribuição de probabilidade uniforme. Logo quando nenhuma informação é conhecida sobre a variável aleatória X, além, obviamente, o vínculo, Eq.(10). A densidade de probabilidade p que maximiza a entropia de Shannon é a uniforme. Nesse caso tem-se:

$$
p_{i}=\frac{1}{n}
$$

Seja agora uma situação um pouco mais geral, quando mais vínculos são prescritos além daquele descrito na Eq. (10). Assim, considera-se que a densidade esteja submetida a "m” outros vínculos escritos na forma:

$$
\sum_{i=1}^{n} p_{i} g_{r i}=a_{r} \quad \text { com } \quad r=1,2,3, \ldots, m
$$

Que podem ser escritos como:

$$
\left\{\begin{array}{c}
\sum_{i=1}^{n} p_{i} g_{1 i}=a_{1} \\
\sum_{i=1}^{n} p_{i} g_{2 i}=a_{2} \\
\vdots \\
\sum_{i=1}^{n} p_{i} g_{m i}=a_{m}
\end{array}\right.
$$

Logo, aplicando o método dos multiplicadores de Lagrange sujeitos aos m vínculos representados na Eq. (11), juntamente ao vínculo representado na Eq. (10), tem-se que:

$$
\begin{aligned}
L\left(p, \lambda_{0}, \lambda_{1}, \lambda_{2}, \ldots, \lambda_{m}\right) & \\
& =-\sum_{i=0}^{n} p_{i} \ln \left(p_{i}\right)-\lambda_{0}\left[\left(\sum_{i=1}^{n} p_{i}\right)-1\right]-\lambda_{1}\left[\left(\sum_{i=1}^{n} p_{i} g_{1 i}\right)-a_{1}\right]-\lambda_{2}\left[\left(\sum_{i=1}^{n} p_{i} g_{2 i}\right)-a_{2}\right]-\ldots \\
& -\lambda_{m}\left[\left(\sum_{i=1}^{n} p_{i} g_{m i}\right)-a_{m}\right]
\end{aligned}
$$

Derivando a Eq. (12) e igualando a zero, escreve-se:

$$
0=\frac{\partial L\left(p, \lambda_{0}, \lambda_{1}, \lambda_{2}, \ldots, \lambda_{m}\right)}{\partial p_{i}}=-\ln \left(p_{i}\right)-1-\lambda_{0}-\sum_{r=1}^{m} \lambda_{r} g_{r i}
$$

Resolvendo esta ultima equação, encontra-se a expressão que nos dá a distribuição de probabilidade $p_{i}$

$$
p_{i}=e^{-\left(1+\lambda_{0}+\lambda_{1} g_{1 i}+\lambda_{2} g_{2 i}+\ldots+\lambda_{m} g_{m i}\right)}
$$

Portanto, substituindo os valores $p_{i}$ encontrados na Eq. (13) nas Eqs (9) e (11), tem-se:

$$
\left\{\begin{array}{c}
\sum_{i=1}^{n} e^{-\left(1+\lambda_{0}+\lambda_{1} g_{1 i}+\lambda_{2} g_{2 i}+\ldots+\lambda_{m} g_{m i}\right)}=1 \\
\sum_{i=1}^{n} e^{-\left(1+\lambda_{0}+\lambda_{1} g_{1 i}+\lambda_{2} g_{2 i}+\ldots+\lambda_{m} g_{m i}\right)} g_{1 i}=a_{1} \\
\sum_{i=1}^{n} e^{-\left(1+\lambda_{0}+\lambda_{1} g_{1 i}+\lambda_{2} g_{2 i}+\ldots+\lambda_{m} g_{m i}\right)} g_{2 i}=a_{2} \\
\vdots \\
\sum_{i=1}^{n} e^{-\left(1+\lambda_{0}+\lambda_{1} g_{1 i}+\lambda_{2} g_{2 i}+\ldots+\lambda_{m} g_{m i}\right)} g_{m i}=a_{m}
\end{array}\right.
$$


Donde é possível observar a partir do conjunto de Eq. (14) um sistema de $(m+1)$ equações e $(m+1)$ incógnitas $\lambda_{0}, \lambda_{1}, \lambda_{2}, \ldots, \lambda_{m}$. Além disso, sua solução leva à caracterização de uma distribuição a partir das informações de conhecimento prévio, denominadas vínculos, em geral estes vínculos são expressos pelos momentos da variável aleatória. Na próxima seção, serão apresentados os resultados preliminares de algumas simulações numéricas aplicando a metodologia previamente apresentada.

\section{RESULTADOS DE SIMULAÇÕES NUMÉRICAS}

Para fins de simulação e caracterização do Princípio da Máxima Entropia (PME), utilizou a caracterização da distribuição normal e gama. A Fig. 3 ilustra a caracterização da distribuição de uma variável aleatória do tipo normal, ilustrando os casos em que a VA possui 2.000, 20.000 e 40.000 elementos. A Tab. 1 mostra os valores determinados pela soma das probabilidades, da média e do desvio padrão da distribuição simulada, valores estes calculados tanto pelo Método da Máxima Entropia quanto via funções disponíveis no software de simulações MATLAB.

Tabela 1: Valores determinados para soma das probabilidades, média e desvio padrão.

\begin{tabular}{c|c|c|c|c|c|c}
\hline & \multicolumn{3}{|c|}{ Método Máxima Entropia } & \multicolumn{3}{c}{ Matlab } \\
\hline $\mathrm{N}$ & $\sum p_{i}$ & $\mu=\sum x_{i} p_{i}$ & $\sigma^{2}=\sum\left(x_{i}-\mu\right)^{2} p_{i}$ & $\sum p_{i}$ & $\mu$ & $\sigma^{2}$ \\
\hline 2000 & 1,0002 & 125,0202 & 9.0001 & 1 & 125 & 9 \\
\hline 20000 & 1,0017 & 125,2187 & 9.0021 & 1 & 125 & 9 \\
\hline 40000 & 1,0378 & 125,7267 & 9,0067 & 1 & 125 & 9 \\
\hline
\end{tabular}

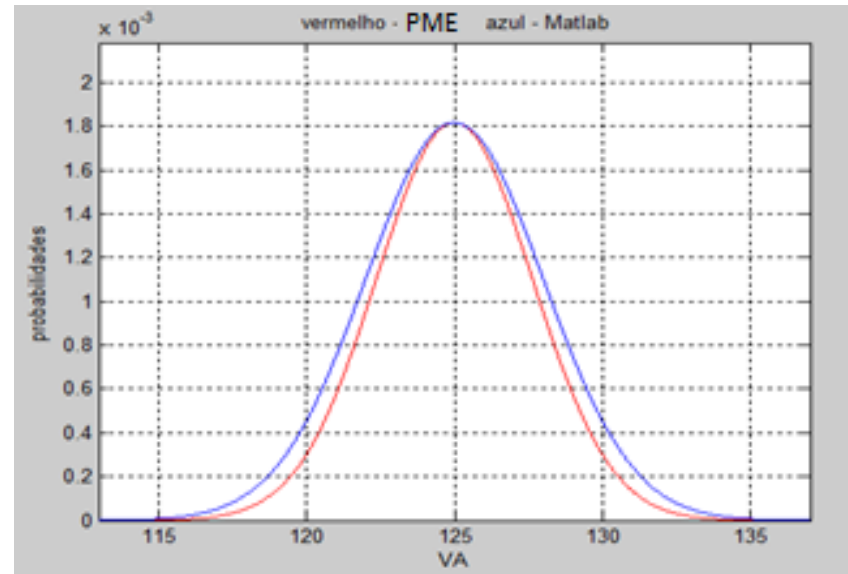

(a) $\mathrm{N}=2000$

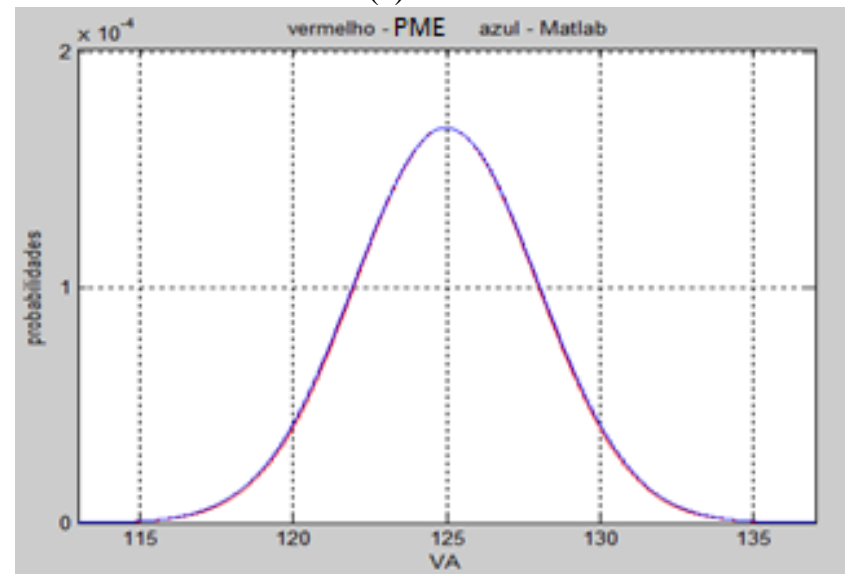

(c) $\mathrm{N}=20000$

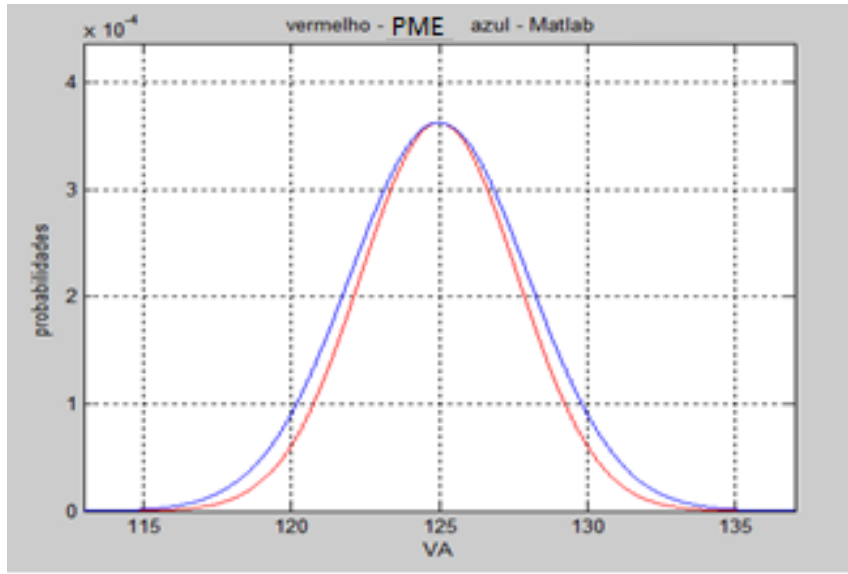

(b) $\mathrm{N}=10000$

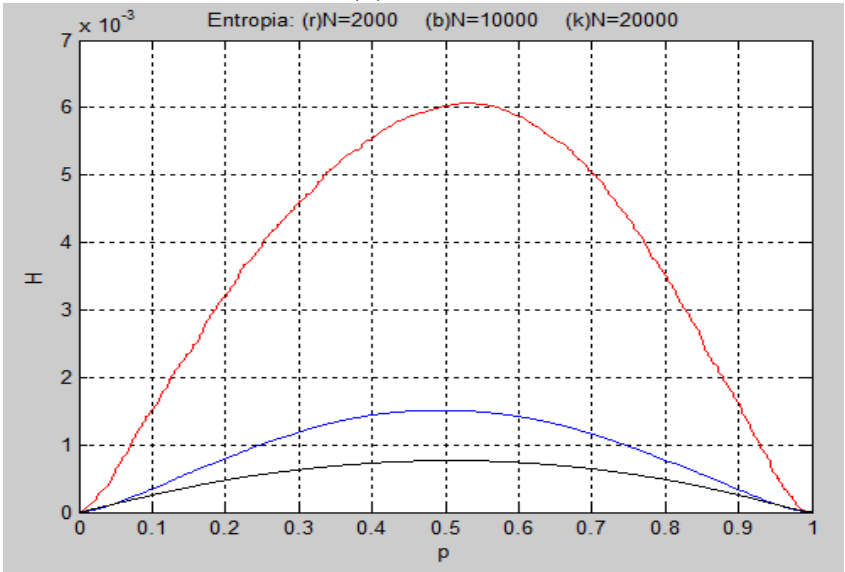

(d) medida de entropia $\mathrm{H}$

Figura 3: Simulações para o caso de uma VA $\operatorname{com} \mu=125 e \sigma=3$.

A Fig. 4 mostra a caracterização de diferentes distribuições a partir das informações disponibilizadas ao Método da Máxima Entropia, na Fig. 4a está ilustrada a caracterização de uma distribuição uniforme quando é conhecido apenas o intervalo de domínio da variável aleatória. No caso da Fig. 4b, a caracterização da distribuição quando são conhecidos o intervalo de domínio da variável aleatória, bem como sua média e variância, já a Fig. 4c buscou-se caracterizar a distribuição de probabilidade quando são conhecidos o domínio da variável e a média, perceba-se que neste caso a distribuição caracterizada é do tipo exponencial e, por fim a Fig. 4d ilustra a caracterização da distribuição, via Método 
da Máxima Entropia, quando se conhece o domínio, a média e a esperança do logaritmo da distribuição. Da mesma forma é possível observar, Fig. 3d a medida de entropia em cada caso simulado, perceba que esta medida é limitada, possuindo um valor máximo, assim como ilustrado na Fig. 2.

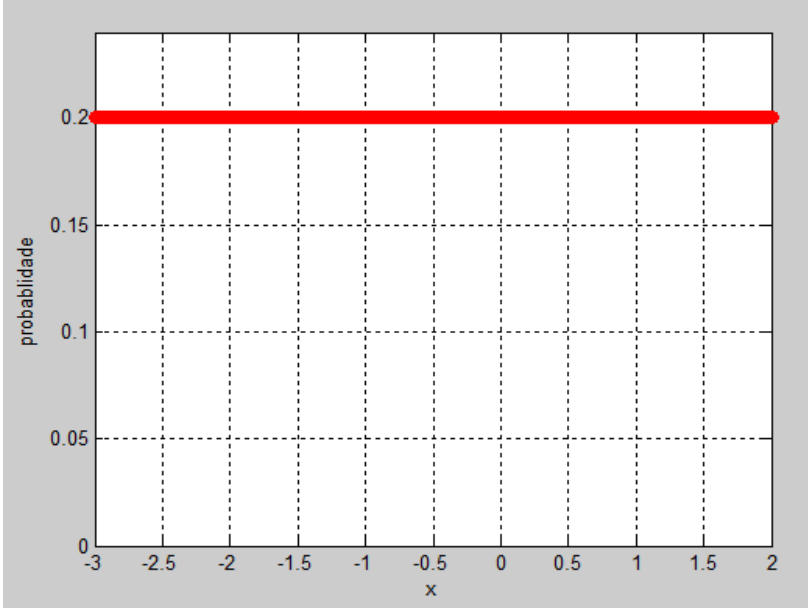

(a) Uniforme

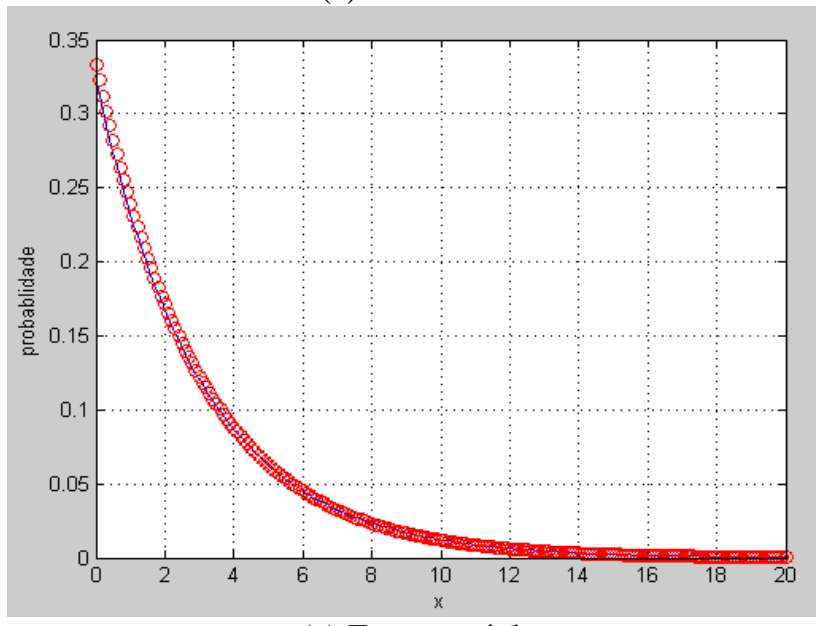

(c) Exponencial

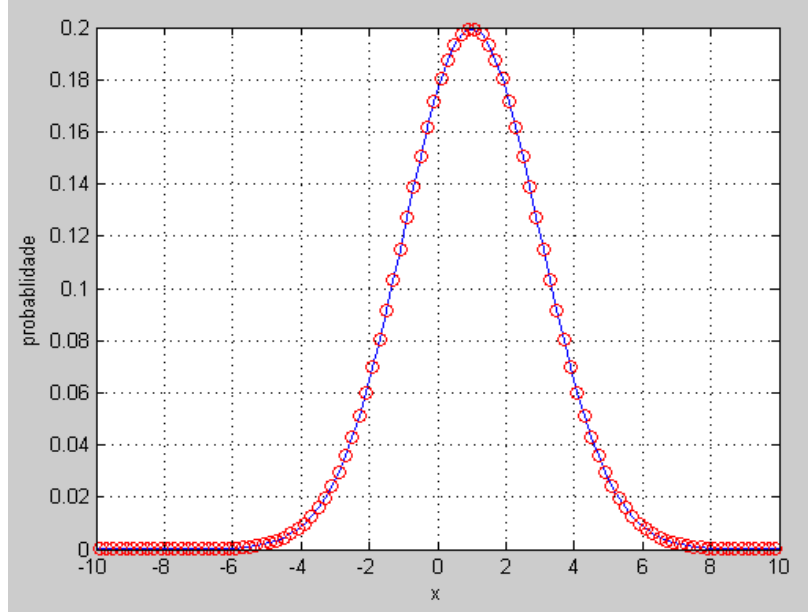

(b) Normal

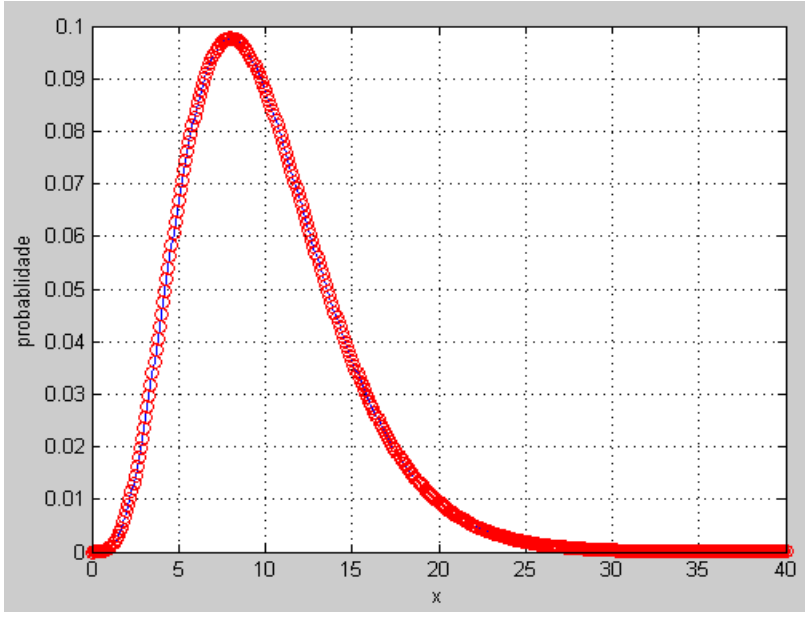

(d) Gama

Figura 4: Caracterização via Método da Máxima Entropia de diferentes distribuições de probabilidades.

Para fins de simulação de um sistema mecânico buscou-se utilizar um sistema conforme ilustrado na Fig. 5, no estabelecimento dos parâmetros incertos, pode-se utilizar tanto a constante elástica da mola, $\mathrm{k}$, bem como sua constante de amortecimento, c, como também a massa, m, do corpo, sabendo-se que as mesmas podem situar-se em intervalos finitos de valores conhecidos, em contrapartida a sua utilização como valores determinísticos para a solução do problema. Porém, para fins de simulação adotou-se o parâmetro de rigidez como parâmetro incerto. Nesse sentido, utilizou-se o Método de Monte Carlo para gerar uma amostra de valores a fim de se obter a faixa de domínio do parâmetro de rigidez da mola, bem como a média e o desvio padrão dessa variável aleatória e, consequentemente de posse dessas informações, utilizar o Método da Máxima Entropia para fins de caracterização da distribuição do parâmetro de rigidez.

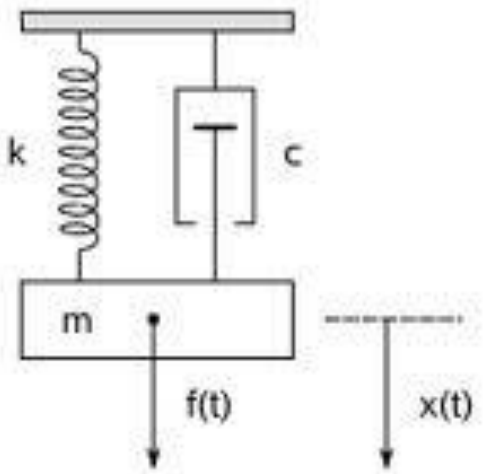

Figura 5: Sistema Massa-Mola-Amortecedor. 
Nesse sentido, a partir do sistema descrito na Fig. 5 é possível encontrar o seguinte modelo matemático, para fins de simulação e obtenção de resultados:

$$
m \ddot{x}+c \dot{x}+k x=f(t)
$$

onde: m: Massa do corpo [kg], c: Coeficiente de amortecimento [N.s/m]; k: Constante Elástica da Mola [N/m]; e $\mathrm{f}(\mathrm{t})$ : Força aplicada $[\mathrm{N}]$.

A Fig. 6, a seguir, ilustra a o gráfico da resposta em frequência caracterizado pela Eq. (15), para uma faixa de frequência de 10 a $45 \mathrm{~Hz}$, perceba que neste gráfico estão representadas quatro curvas sendo três delas determinadas pelo Método de Monte Carlo, as quais estão descritas pelas cores azul(b), preta(k) e vermelha(r), sendo a curva na cor verde(g) caracterizada pelo Princípio de Máxima Entropia, quando se conhece a faixa de domínio, a média e o desvio padrão da distribuição. Observe que as curvas nas cores preta e verde são praticamente idênticas (vide foco identificado pelos círculos na Fig. 6b), o que evidencia o Princípio de Máxima Entropia como um excelente método para caracterização da distribuição neste caso. Logo abaixo, na Tab. 2 está descrito os parâmetros utilizados no Método da Máxima Entropia, para fins de caracterização da distribuição aqui representada para fins de obtenção da distribuição associada ao parâmetro de rigidez da mola (k).

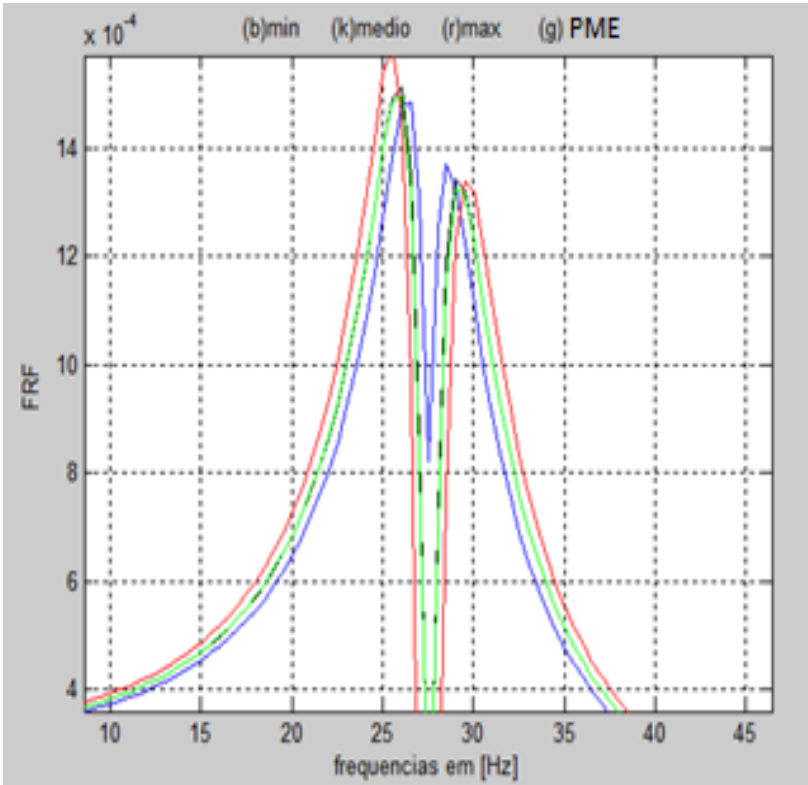

(a)

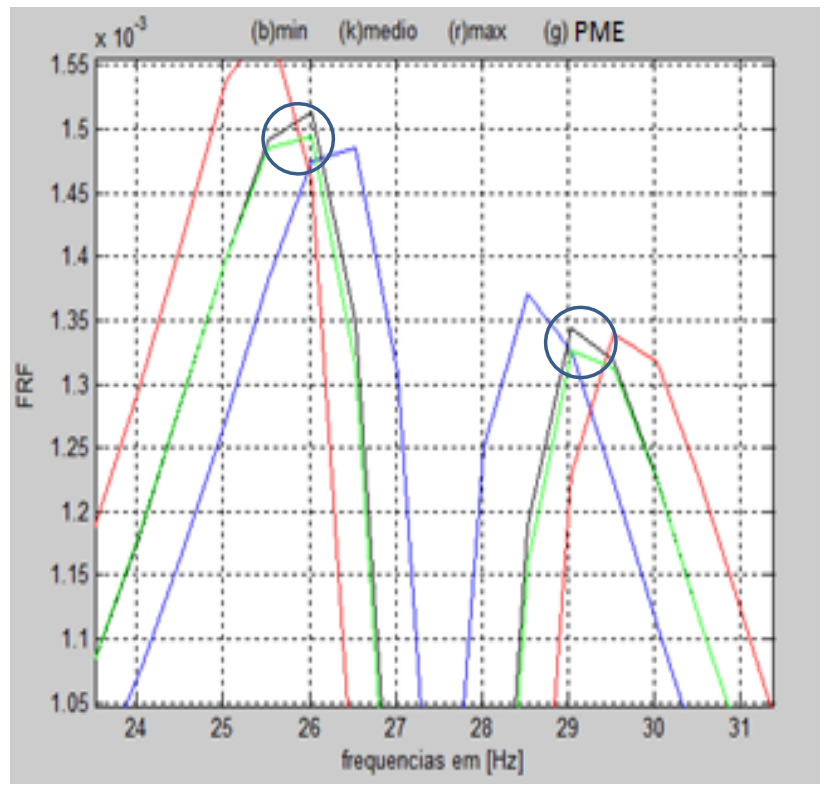

(b)

Figura 6: Gráfico FRF utilizando o MMC e PME.

Tabela 2: Dados de simulação e obtidos via PEM.

\begin{tabular}{|c|c|c|c|c|c|c|}
\hline & \multicolumn{3}{|c|}{ Método Máxima Entropia } & \multicolumn{3}{|c|}{ Matlab (R2010a) } \\
\hline $\mathrm{N}$ & $\sum p_{i}$ & $\mu=\sum_{[\mathrm{N} / \mathrm{m}]} x_{i} p_{i}$ & $\sigma^{2}=\sum_{\left[(\mathrm{N} / \mathrm{m})^{2}\right]}\left(x_{i}-\mu\right)^{2} p_{i}$ & $\sum p_{i}$ & $\begin{array}{c}\mu \\
{[\mathrm{N} / \mathrm{m}]}\end{array}$ & $\begin{array}{c}\sigma^{2} \\
{\left[(\mathrm{~N} / \mathrm{m})^{2}\right]}\end{array}$ \\
\hline 1000 & 1,0000 & 3000,2 & 60,3 & 1 & 3 & 60,2 \\
\hline
\end{tabular}

\section{CONCLUSÕES GERAIS}

Neste trabalho, procurou-se de forma rápida ilustrar a caracterização dos fundamentos do Princípio da Máxima Entropia, buscando ilustrar, de forma objetiva, a razão da utilização da medida de entropia H, assim como definida por Shannon e caracterizada por Jaynes, no sentido de caracterizar uma distribuição de probabilidade sem que se conheça, previamente, os elementos da variável aleatória, bastando apenas conhecer uma ou mais informações da variável aleatória, tais como domínio e momentos de primeira e segunda ordem.

A partir dos expostos ao longo do texto é possível perceber que a medida de entropia é uma medida restrita a uma faixa de valores e, por conseguinte, tornando o método robusto e completo para a caracterização de uma determinada distribuição de probabilidades, a partir de informações prévias, neste mesmo sentido é possível dizer que o método é vulnerável quanto às informações subjugadas.

Por fim, a partir das figuras apresentadas é possível perceber a robustez do Método da Máxima Entropia, em especial quando é analisada a Fig. 6, onde percebe-se claramente uma boa aproximação da distribuição determinada. 


\title{
5. AGRADECIMENTOS
}

Às Agencias de fomento à pesquisa, CAPES, CNPQ e FAPEMIG pelo apoio financeiro, revertidos em bolsas de estudos e projetos de pesquisas desenvolvidos pelo Programa de Pós-graduação em Engenharia Mecânica da Universidade Federal de Uberlândia, bem como à Universidade Estadual de Goiás pelo apoio financeiro disponibilizado via do Programa de Auxílio a Eventos (Pró-Eventos).

\section{REFERÊNCIAS}

Cattani, M. e Bassalo J. M. F. 2008. Entropia, reversibilidade, irreversibilidade, equação de transporte e teorema H de Boltzmann e o teorema do retorno de Poincaré. Revista Brasileira de Ensino de Física, v. 30, n² 2, pp. 2301.

Jaynes, E. T. 1957. Information Theory and Statistical Mechanics. The Physical Review, Vol. 106, $\mathrm{n}^{0 .}$, (pp. 620-630).

Koçer, B. 2010. Vibration Fatigue Analysis of Structures under Broadband Excitation, Ph.D. thesis submitted to the graduate school of natural and applied sciences of Middle East Technical University.

Lambert, S., Pagnacco, E., Khalij, L., Leo, D.J. 2010. A Probabilistic Model for the Fatigue Reliability of Structures Under Random Loadings with Phase Shift Effects, Int. Journal of Fatigue, Vol. 32, (pp. 463-474).

Lima, A.M.G., Rade, D.A. and Bouhaddi, N. 2010. Stochastic Modeling of Surface Viscoelastic Treatments Combined with Model Condensation Procedures", Shock and Vibration, Vol. 17, (pp. 429-444).

Sampaio, R., Cataldo, E. 2010. Comparing two strategies to model uncertainties in structural dynamics", Shock and Vibration, Vol. 14, (pp. 171-186).

Shannon, C. E. 1948. A Mathematical Theory of Communication. The Bell System Technical Journal, Vol. 27, (pp. $379-423 ; 623-656$ ).

\section{RESPONSABILIDADE AUTORAL}

Os autores são os únicos responsáveis pelo conteúdo deste trabalho.

\section{Numerical-computer study of Maximum Entropy Method in Analysis of Parametric Uncertainty in Systems Engineering}

\author{
Marcelo Henrique Belonsi - marcelobelonsi@bol.com.br ${ }^{1}$ \\ Antonio Marcos Gonçalves de Lima - amglima@ mecanica.ufu.br ${ }^{2}$ \\ Romer Gonçalves - romer.engenharia@gmail.com²
}

\footnotetext{
${ }^{1}$ Universidade Estadual de Goiás, Departamento de Matemática, Rua 14, 625, Jardim América, Morrinhos-GO. ${ }^{2}$ Universidade Federal de Uberlândia, Faculdade de Engenharia Mecânica, João Naves de Ávila, 2121, Santa Monica, Uberlândia-MG.
}

\begin{abstract}
The Maximum Entropy Principle has its development between the years 1844 and 1906, with the publications of the results obtained by the Boltzmann's Transport Equation and the Bolstzmann's H Theorem, whose objectives were to determine the temporal variation from a probability distribution function, furthermore the characterization of a metric to the Boltzmann's Transport Equation. However, under the possibility to extend the concept of entropy to physical systems, Shannon has employed Boltzmann's jobs research to the search of the measurement of entropy, which would characterize the probability distribution of a random variable. In that sense, this job are dedicated to the theoretical formalization about the principle and its properties from Shannon's work, aiming to characterize its formulas and hence its validity and effectiveness in the characterization of the probability densities for the application in engineering problems. In that way, it will be highlighted all the theoretical developments to the characterization of the essential mathematical relations of the Maximum Principle of Entropy aiming its applicability to dynamic systems in general and yet show how the mathematic of probability is present in such various types application, especially in those concerned to mechanical engineering.
\end{abstract}

Keywords: Quantification of uncertainties; Maximum Entropy Principle; Mathematics; Dynamics. 